Pacific Journal of Mathematics

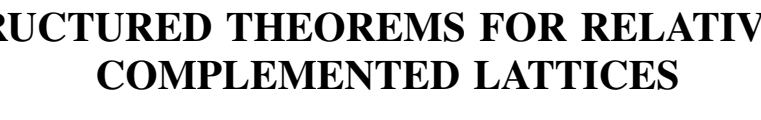




\title{
STRUCTURED THEOREMS FOR RELATIVELY COMPLEMENTED LATTICES
}

\author{
J. E. MCLAUGHLIN
}

Introduction. In a previous paper [3] a study was made of the projectivities between the points of a simple relatively complemented lattice of finite dimension. It was shown that for a given dimension there is an upper bound for the number of transposes required to establish the projectivities between the points. The examples given in which this upper bound is attained have a particularly simple structure - they are closely related to a direct union. We shall prove here some general structure theorems for relatively complemented lattices and then apply these to the case of maximal projectivities.

The notation will be that of [3]. The lattice $L$ to which we refer is always relatively complemented.

1. Structure Theorems. Our arguments depend heavily upon the simplicity or indecomposability [2] of $L$, and it is convenient to have the following characterization of a direct union:

THEOREM 1.1. If $L$ has dimension $n$, and $a, b$ are two elements of $L$, then $L \cong a / z \vee b / z$ if and only if

(1) $\rho(a)+\rho(b) \leq n$, and

(2) $p \subseteq a$ if and only if $p \nsubseteq b$ for all points $p \in L$.

Proof. Certainly if $L \cong a / z \vee b / z$, conditions (1) and (2) will hold. Suppose (1) and (2) hold in $L$. We shall proceed by induction on $n$. The theorem is true when $n=1,2$. Suppose it is true for all lattices of dimension less than $n$, but $L \neq a / z \vee b / z$.

It is clear that

$$
x=(a \cap x) \cup(b \cap x)
$$

for all $x \in L$. Consider the mapping

Received March 31, 1952.

Pacific J. Math. 3 (1953), 197-208 


$$
x \rightarrow \sigma x=(a \cap x, b \cap x) \in a / z \vee b / z
$$

Yow $x \supseteq y$ if and only if

$$
a \cap x \supseteq a \cap y \text { and } b \cap x \supseteq b \cap y
$$

and the latter occurs if and only if $\sigma x \supseteq \sigma y$. Hence $L$ is isomorphic, as a partially ordered set, to a subset of $a / z \vee b / z$, where

$$
\sigma u=(a, b), \sigma z=(z, z) \text {. }
$$

These remarks show that if any two elements $a, b$ of $L$ satisfy (2), we must have

$$
\rho(a)+\rho(b) \geq n
$$

If $L \neq a / z \vee b / z$, there are points $p \subseteq a$ and $q \subseteq b$ such that $p / z \quad P_{2} q / z$. Hence there is a maximal element $m$ such that $m \neq p, m \nmid q$. Then $s_{1}$ and $s_{2}$ exist with

$$
a>s_{1} \supseteq m \cap a, b>s_{2} \supseteq m \cap b .
$$

Furthermore,

$$
a \cup s_{2}=b \cup s_{1}=u \text {. }
$$

Let $u=x_{0}>x_{1}>\cdots>x_{n-1}>x_{n}=z$ be a complete chain in $L$. This chain maps onto

$$
\sigma u=(a, b)>\sigma x_{1}>\cdots>\sigma x_{n-1}>\sigma x_{n}=(z, z) .
$$

Either (i) $\sigma x_{1}=\left(a, t_{2}\right)$, where $b>t_{2}$, or (ii) $\sigma x_{1}=\left(t_{1}, b\right)$, where $a>t_{1}$. Suppose the former is true. The points of $x_{1}$ are in either $a$ or $t_{2}$, but not both. Then $a$ and $t_{2}$ satisfy $(2)$ in $x_{1} / z$, and since

$$
\rho\left(x_{1}\right)=n-1
$$

we have

$$
\rho(a)+\rho\left(t_{2}\right) \geq n-1 \text {. }
$$

But

$$
\rho(a)+\rho(b) \leq n \text {, so } \rho\left(t_{2}\right)=\rho(b)-1
$$

Then by the induction hypothesis, $x_{1} / z \cong a / z \vee t_{2} / z$. This gives the exist- 
ence of a chain from $s_{1}$ through $a$ to $u$ of length $1+\rho(b)-1+1$, or $\rho(b)+1$. By Lemma 3.6 of [3], there is a chain from $b$ to $z$ of length at least $\rho(b)+1$, which is a contradiction. A similar contradiction arises if $\sigma x_{1}=\left(t_{1}, b\right)$. There fore $L \cong a / z \vee b / z$, and thus the theorem is proved.

The following theorem gives more information about the quotient lattices $a_{p}^{k} / z$ introduced in Lemma 3.5 of [3].

THEORE 1.2. Let $L$ be simple of dimension $n>1$. If $p$ is any point and $k$ is a nonnegative integer such that $h<[(n+1) / 2]$, then $a_{p}^{k} / z$ has dimension at least $2 k+1$.

Proof. The theorem is true when $k=0$. Suppose it is true for all $k$ less than the one in which we are interested. Then $a_{p}^{k-1}$ has dimension at least $2 k-1$, and $a_{p}^{k} \supseteq a_{p}^{k-1}$. If $a_{p}^{k}=u$, we are through, so assume $u \supset a_{p}^{k}$. Then there is a point $s \in L$ with $s \notin a_{p}^{k}$, but $s / z P_{2} t / z$ for some $t \in C_{p}^{k}$. Hence there is a maximal element $m$ such that $m \nsupseteq s, m \nsupseteq a_{p}^{k}$. Since $s \in C_{p}^{k}$, we have $m \supseteq a_{p}^{k-1}$. Therefore $a_{p}^{k} \supset a_{p}^{k-1}$, and the dimension of $a_{p}^{k} / z$ is at least $2 k$. Suppose $\operatorname{dim}\left(a_{p}^{k} / z\right)=2 k$. I et $b$ be the join of all points of $L$ which are not in $a_{p}^{k}$. All of these points are in $x_{p}^{k}=\cap M_{p}^{k}$, where

$$
u_{p}^{k} \equiv\left\{m \in L \mid u>m \nsupseteq a_{p}^{k-1}\right\} .
$$

( See proof of Lemma 3.5 of [3].) Hence $x_{p}^{k} \supseteq b$ and $b \cap a_{p}^{k}=z$. The latter follows from the assumption $\operatorname{dim}\left(a_{p}^{k} / z\right)=2 k$, since, by Theorem 3.1 of [3] for any point $q$ we would have $q \subseteq a_{p}^{k}$ if and only if $q \in C_{p}^{k}$. On the other hand, it is shown, in the proof of Lemma 3.5 of [3], that $q \in C_{p}^{k}$ if and only if $q \nsubseteq x_{p}^{k}$.

Since $L$ is simple, there exists an $x$ such that

$$
u>x, x \neq a_{p}^{k}, x \neq b .
$$

But $x \nsupseteq b$ implies $x \supseteq a_{p}^{k-1}$. Then

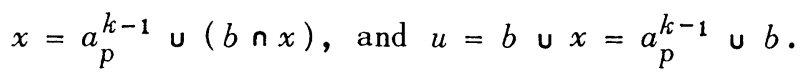

Hence if $u>m$ we have $m \supseteq a_{p}^{k-1}$, if and only if $m \nsupseteq b$. Therefore $a_{p}^{k}, a_{p}^{k-1}$, and $b$ satisfy the conditions of Lemma 3.6 of [3], and there exists a chain of length at least $2 k$ from $u$ to $b$. Then

$$
\rho(b) \leq n-2 k, \text { so } \rho\left(a_{p}^{k}\right)+\rho(b) \leq n .
$$

But by Theorem 1.1 we would have $L=a_{p}^{k} / z \vee b / z$, contrary to the simplicity of $L$. Therefore $\rho\left(a_{p}^{k}\right) \geq 2 k+1$ for all $k<[(n+1) / 2]$. 
Let $\Re$ denote the partially ordered subset of $L$ consisting of $u$, the maximal elements, the points, and $z$. Let $\beta_{\nu}$ be the normal completion of $q_{\beta}$. Consider the mapping $A \longrightarrow \cup A$ from $\beta_{\nu}$ into $L$. ( $A$ is a normally closed subset of $L$.) If $A \supseteq B$, then $\cup A \supseteq \cup B$. Suppose $\cup A \supseteq \cup B$; then $x \in A^{*}$ implies $x \supseteq a$, all $a \in A$ implies $x \supseteq \cup A$, so $x \supseteq \cup B$, and hence $x \supseteq b$ all $b \in B$ and $x \in B^{*}$; therefore $A^{*} \subseteq B^{*}$, so $\left(A^{*}\right)_{*} \supseteq\left(B^{*}\right)_{*}$, or $A \supseteq B$. Thus the mapping is order preserving both ways.

Suppose $a \in L, a \neq u, a \neq z$. Set

$$
\begin{aligned}
& P(a) \equiv\{p \in \Re \mid a \supseteq p>z\}, \\
& M(a) \equiv\{m \in \Re \mid u>m \supseteq a\} .
\end{aligned}
$$

Now $x \supseteq p$, all $p \in P(a)$, if and only if $x \supseteq a$, so $M(a) \subseteq(P(a))^{*}$. Also $P(a) \subseteq\left(P(a)^{*}\right)_{*}$. Suppose $y \in\left(P(a)^{*}\right)_{*}$; then $y \subseteq x$, all $x \in P(a)^{*}$ implies $y \subseteq m$, all $m \in M(a)$ implies $y \subseteq a$. Suppose $a^{\prime} \supseteq y$, all $y \in\left(P(a)^{*}\right)_{*}$; then $a^{\prime} \supseteq p$, all $\dot{p} \in P(a)$ implies $a^{\prime} \supseteq a$, so $a=U\left(P(a)^{*}\right)_{*}$. If $a=u$, then $a=U(u)$; if $a=z$ then $a=\mathrm{U}(z)$. (Here $(x)$ denotes the principal ideal generated by $x_{0}$ ) Hence each $a \in L$ has an inverse image under the above mapping, and $\Re_{\nu} \cong L$; see [2]. This proves the following:

THEOREM 1.3. The structure of $L$ is completely determined by the structure of $\Re$.

REMARK. From the nature of the proof it is seen that the above theorem will be true for any lattice each of whose elements is a join of points and the meet of maximal elements.

2. Lattices with maximal projectivities. In this section we shall study simple lattices of odd dimension in which there occurs a maximal projectivity. We shall show that these lattices are quite close to a direct union in the sense that their structure can be completely described in terms of sublattices. Throughout this section $L$ will be a simple lattice of dimension $2 n+1$, and $p, q$ are two points in $L$ such that $p / z P q / z$ requires $2 n+2$ transposes. Then we have:

THEOREM 2.1. If $k \leq n$, the following statements are true:

(1) $\rho\left(a_{p}^{k}\right)=2 k+1, \rho\left(a_{q}^{n-k}\right)=2 n-2 k+1$;

(2) $x_{p}^{k}=a_{q}^{n-k}, x_{q}^{n-k}=a_{p}^{k}$;

(3) $a_{p}^{k} / z$ has a maximal projectivity if and only if $a_{q}^{n-k} / z$ has a maximal projectivity; 
(4) if $a_{p}^{k} / z$ has a maximal projectivity then $a_{p}^{k} \cap a_{q}^{n-k}=r>z$, otherwise $a_{p}^{k} \cap a_{q}^{n-k}=z$

Proof. Note that $s \in C_{. q}^{n-k}$ implies $s \notin C_{p}^{k}$ implies $s \subseteq x_{p}^{k}$ implies $a_{q}^{n-k} \subseteq x_{p}^{k}$. Suppose there is a maximal element $m$ such that $m \supseteq a_{p}^{k-1}, m \supseteq x_{p}^{k}$. If $m / z$ is simple, we contradict the assumption of a maximal projectivity between $p / z$ and $q / z$, since $\rho(m) \leq 2 n$. Write

$$
m / z=L_{1} \vee L_{2} \vee \ldots \vee L_{\nu}
$$

where the $L_{i}$ are simple nontrivial quotient lattices, and $\nu>1$. Now $a_{q}^{n-k} / z$ and $a_{p}^{k-1} / z$ are both simple; if they are in the same $L_{i}$, we again contradict our maximal projectivity assumption. Hence they are in different components and we must have

$$
\rho\left(a_{p}^{k-1}\right)+\rho\left(a_{q}^{n-k}\right) \leq 2 n
$$

By Theorem 1.2,

$$
\rho\left(a_{p}^{k-1}\right) \geq 2 k-1 ; \rho\left(a_{q}^{n-k}\right) \geq 2 n-2 k+1 .
$$

Therefore

$$
\rho\left(a_{q}^{n-k}\right)=2 n-2 k+1
$$

The elements $a_{p}^{k-1}$ and $a_{q}^{n-k}$ are in different $L_{i}$, so

$$
\rho\left(a_{p}^{k-1} \cup a_{q}^{n-k}\right)=\rho\left(a_{p}^{k-1}\right)+\rho\left(a_{q}^{n-k}\right) \geq 2 n
$$

and hence

$$
m=a_{p}^{k-1} \cup a_{q}^{n-k} \text { or } m / z=a_{p}^{k-1} / z \vee a_{q}^{n-k} / z
$$

Now let $s>z, s \subseteq x_{p}^{k}$. Then $s \notin C_{p}^{k}$, so $s \nsubseteq a_{p}^{k-1}$. But $m \supseteq x_{p}^{k}$, so $m \supseteq s$, and therefore $s \subseteq a_{q}^{n-k}$. This shows that $x_{p}^{k} \subseteq a_{q}^{n-k}$, and hence $x_{p}^{k}=a_{q}^{n-k}$. Thus we have shown that if $a_{p}^{k-1} \cup x_{p}^{k} \neq u$, then $x_{p}^{k}=a_{q}^{n-k}$ and $\rho\left(a_{q}^{n-k}\right)=2 n-2 k+1$.

Suppose $a_{p}^{k-1} \cup x_{p}^{k}=u$. Then for each maximal element $m, m \supseteq a_{p}^{k-1}$ if and only if $m \nsupseteq x_{p}^{k}$. We have $\rho\left(a_{p}^{k-1}\right) \geq 2 k-1$, so $\operatorname{dim}\left(u / a_{p}^{k-1}\right) \leq 2 n+2-2 k$. Since $L$ is simple, $\operatorname{dim}\left(u / x_{p}^{k}\right) \geq 2 k$, by Theorem 1.1. Hence $\rho\left(x_{p}^{k}\right) \leq 2 n-$ $2 k+1$. But $x_{p}^{k} \supseteq a_{q}^{n-k}$, and $\rho\left(a_{q}^{n-k}\right) \geq 2 n-2 k+1$. Hence, in all cases, $x_{p}^{k}=a_{q}^{n-k}$ and $\rho\left(a_{q}^{n-k}\right)=2 n-2 k+1$. By a similar argument, $x_{q}^{n-k}=a_{p}^{k}$ and $\rho\left(a_{p}^{k}\right)=2 k+1$. This demonstrates $(1)$ and $(2)$. 
Suppose $r>z, r \subseteq a_{p}^{k}$ such that $r / z P p / z$ requires $2 k+2$ transposes. Now $r \notin C_{p}^{k}$ implies $r \subseteq x_{p}^{k}=a_{q}^{n-k}$. Furthermore, $r \subseteq a_{p}^{k}=x_{q}^{n-k}$ implies $r \notin C_{q}^{n-k}$ implies that $r / z P q / z$ requires $2 n-2 k+2$ transposes. The argument is symmetric in $p$ and $q$, and this proves (3).

Suppose $s>z$ and $s / z P p / z$ requires $2 n+2$ transposes. Then $x_{p}^{n}=a_{s}^{0}=$ $s=a_{q}^{0}=q$, so there is at most one point $q$ such that $p / z P q / z$ requires $2 n+2$ transposes. This shows that the $r$ in the preceding paragraph, if it exists, is unique, and we have (4).

Wive are now in a position to characterize the maximal elements of $L$ in terms of the structure of $a_{p}^{k} / z$ and $a_{q}^{n-k} / z$. When we know these maximal elements, we will know the structure of $L$, by Theorem 1.3. First we prove two useful lemmas.

L.FMMA 2.1. There is a chain of length $2 n+1$ through $a_{p}^{k}$.

Suppose $a_{p}^{k} \cup a_{q}^{n-k-1}=u$. Then the maximal elements of $L$ are in two disjoint classes - those containing $a_{p}^{k}$ and those containing $a_{q}^{n-k-1}$; and by Theorem 1.1 ,

$$
\operatorname{dim}\left(u / a_{p}^{k}\right)+\operatorname{dim}\left(u / a_{q}^{n-k-1}\right)>2 n+1 .
$$

But

$$
\begin{aligned}
& \operatorname{dim}\left(u / a_{p}^{k}\right) \leq 2 n+1-(2 k+1) \\
& \operatorname{dim}\left(u / a_{q}^{n-k-1}\right) \leq 2 n+1-(2 n-2 k-1) .
\end{aligned}
$$

Hence $\operatorname{dim}\left(u / a_{p}^{k}\right)=2 n-2 k$.

Suppose $u>m \supseteq a_{p}^{k} \cup a_{q}^{n-k-1}$. Now $m / z$ is not simple, since $\rho(m) \leq 2 n$ and $m \supseteq p, m \supseteq q$. Suppose

$$
m / z=L_{1} \vee L_{2} \vee \ldots \vee L_{\nu}
$$

where $\nu>1$. Then $a_{p}^{k} / z$ and $a_{q}^{n-k-1} / z$ are in different components and again there is a chain from $a_{p}^{k}$ to $u$ of length at least $2 n-2 k$ since $\rho\left(a_{q}^{n-k-1}\right)=2 n-$ $2 k-1$. This proves the lemma.

LEMMA 2.2. If $s>z, a \nsupseteq s, b \nsupseteq s, b u t a \cup b \supseteq s$, then there are points $s_{1} \subseteq a$, $s_{2} \subseteq b$ such that $s_{1} / z P_{2} s / z$ and $s_{2} / z P_{2} s / z$.

Let $s \cup b>x \supseteq b$, and let $x^{\prime}$ be a relative complement of $s \cup b$ in $a \cup b / x$ such that $a$ u $b>x^{\prime}$. Then $x^{\prime} \nsupseteq a, x^{\prime} \nsupseteq s$; hence $x^{\prime} \nsupseteq s_{1}$, for some point $s_{1} \subseteq a$. Therefore $s / z T a \cup b / x^{\prime} T s_{1} / z$. Similarly we can show the existence of $s_{2}$. 
proving the lemma.

LENNA 2.3. The following relation holds: $\operatorname{din}\left(a^{k} / a_{p}^{k-1}\right)=2$.

For since $L$ is simple there is a maximal $m_{0}$ such that $m_{0} \nsupseteq a_{p}^{k}, m_{0} \nsupseteq a_{q}^{n-k}$. Then $m_{0} \supseteq a_{p}^{k-1}, m_{0} \supseteq a_{q}^{n-k-1}$. Assume $a_{p}^{k}>a_{p}^{k-1}$. Then $m_{0} n a_{p}^{k}=a_{p}^{k-1}$. Set $w=a_{q}^{n-k} n m_{0}$. Then $y$ exists such that $a_{q}^{n-k}>y \supseteq w$. Since $m_{0}=a_{p}^{k-1} u w$, we have $u=a_{p}^{k-1} \cup y=w \cup a_{p}^{k}$. Since there is a chain of length $2 k$ from $a_{q}^{n-k}$ to $u$, there exists a maximal $m$ such that $m \neq a_{q}^{n-k}$ and such that there exists a chain of length at least $2 k$ from $m$ to $y$. Now $m \neq a_{p}^{k}$ since $a_{p}^{k}$ u $y=u$. But $m \supseteq a_{p}^{k-1}$ and $m / z=a_{p}^{k-1} / z \vee y / z$ in contradiction with the length of the chain from $y$ to $m$. llence $a^{k} \ngtr a_{p}^{k-1}$, and we must have $\operatorname{dim}\left(a_{p}^{k} / a_{p}^{k-1}\right)=2$.

COROLLARY. The following relation holds:

$$
\operatorname{dim}\left(a_{q}^{n-k} / a_{q}^{n-k-1}\right)=2
$$

This follows by symmetry.

3. Maximal elements when $a_{p}^{k} n a_{q}^{n-k} \neq z$. The following theorem gives the possibilities for maximal elements when $a_{p}^{k} / z$ and $a_{q}^{n-k} / z$ each have a maximal projectivity. We assume throughout that $1 \leq k \leq n-1$.

THEOREM 3.1. Let $a_{p}^{k} \cap a_{q}^{n-k}=r>z$, and let $u>m$. If $m \supseteq r$, either

(1) $m \supseteq a_{p}^{k}$ and $a_{q}^{n-k}>m \cap a_{q}^{n-k}$,

or

( 2) $a_{p}^{k}>m \cap a_{p}^{k}$ and $m \supseteq a_{q}^{n-k}$.

If $m \nsupseteq r$, then $a_{p}^{k}>a_{p}^{k} \cap m$ and $a_{q}^{n-k}>a_{q}^{n-k} \cap m$.

Proof. Let $u>m \supseteq r$, and suppose $m \nsupseteq a_{p}^{k}, m \nsupseteq a_{q}^{n-k}$. Then $m \supseteq a_{p}^{k-1}$, and $m \supseteq a_{q}^{n-k-1}$, for otherwise we would not have a maximal projectivity in $L$. For the same reason, we have $r \nsubseteq a_{p}^{k-1}, r \nsubseteq a_{q}^{n-k-1}$. Then since

$$
\begin{array}{ll}
\rho\left(a_{p}^{k-1}\right)=2 k-1, & \rho\left(a_{p}^{k}\right)=2 k+1, \\
\rho\left(a_{q}^{n-k-1}\right)=2 n-2 k-1, & \rho\left(a_{q}^{n-k}\right)=2 n-2 k+1,
\end{array}
$$

we must have

$$
a_{p}^{k}>m \cap a_{p}^{k}=r \cup a_{p}^{k-1} \text { and } a_{q}^{n-k}>m \cap a_{q}^{n-k}=r \cup a_{q}^{n-k-1}
$$

Hence 


$$
m=\left(r \cup a_{p}^{k-1}\right) \cup\left(r \cup a_{q}^{n-k-1}\right) \text { and } u=a_{p}^{k} \cup m=a_{p}^{k} \cup a_{q}^{n-k-1} .
$$

Similarly, $u=a_{q}^{n-k} \cup a_{p}^{k-1}$.

ijy I.emma 2.1, there is a chain from $a_{p}^{k}$ to $u$ of length $2 n-2 k$. Since $L$ is relatively complemented, it is easy to see that $v$ exists such that $u>v, v \cap a_{p}^{k}=$ $r \cup a_{p}^{k-1}$, and there is a chain from $r \cup a_{p}^{k-1}$ to $v$ of length at least $2 n-2 k$. There is an $s \in C_{p}^{k}$ such that $s \nsubseteq a_{p}^{k-1} \cup r$. Hence $s \nsubseteq v$, and this implies $v \supseteq a_{q}^{n-k-1}$. Therefore $v \supseteq m$ and $v=m$. Then by Theorem 1.1 ,

$$
m / z=a_{p}^{k-1} \text { } r / z \vee a_{q}^{n-k-1} / z
$$

but this contradicts the existence of a chain from $a_{p}^{k-1} \cup r$ to $m$ of length $2 n-$ $2 k$. Hence we must have either $n \supseteq a_{p}^{k}$, or $m \supseteq a_{q}^{n-k}$.

Suppose $m \supseteq a_{q}^{n-k}$, but $a_{p}^{k}>x \supset m \cap a_{p}^{k}$. Let $y$ be a relative complement of $x$ in $a_{p}^{k} / a_{p}^{k} \cap m$. Then $y \supset a_{p}^{k} \cap m$, since $a_{p}^{k}>x$. lience $m \nsupseteq x, m \nsupseteq y$, so

$$
x \cup a_{q}^{n-k}=y \cup a_{q}^{n-k}=u \text {. }
$$

Since

$$
\rho\left(a_{p}^{k}\right)=\rho\left(a_{p}^{k-1}\right)+2 \text { and } r \cup a_{p}^{k-1} \supset a_{p}^{k-1}
$$

it follows that $m \nsupseteq a_{p}^{k-1}$. Hence either $x \nsupseteq a_{p}^{k-1}$ or $y \nsupseteq a_{p}^{k-1}$. Suppose the latter is the case. Then there is an $s \in C_{p}^{k-1}$ such that $s \nsubseteq y$. But $s \subseteq u=y u a_{q}^{n-k}$. Hence, by Lemma $2.2, s / z P_{2 n-2 k+2} q / z$ and $p / z P_{2 n} q / z$ contrary to our assumption of a maximal projectivity between $p / z$ and $q / z$. A similar contradiction arises if $x \nsupseteq a_{p}^{k-1}$. Hence $a_{p}^{k}>a_{p}^{k} n m$. The roles of $p$ and $q$ are symmetric, so if $m \supseteq a_{p}^{k}$, then $a_{q}^{n-k}>m \cap a_{q}^{n-k}$.

Now let $u>m \nsupseteq r$. Since $m \nsupseteq r$, we have $m \supseteq a_{p}^{k-1}$ and $m \supseteq a_{q}^{n-k-1}$. Suppose

$$
a_{p}^{k}>x>a_{p}^{k-1}=a_{p}^{k} \cap m \text { and } a_{q}^{n-k}>y>a_{q}^{n-k-1}=a_{q}^{n-k} \cap m .
$$

Let $x^{\prime}$ be a relative complement of $x$ in $a_{p}^{k} / a_{p}^{k-1}$. Suppose $x^{\prime} \nsupseteq r$, and let $x^{\prime \prime}$ be a relative complement of $a_{p}^{k}$ in $u / x^{\prime}$. Since $a_{p}^{k}>x^{\prime}$, we can assume $u>x^{\prime \prime}$. Now $x^{\prime \prime} f r$, so $x^{\prime \prime} \supseteq a_{q}^{n-k-1}$. Hence $x^{\prime \prime}=m$, contrary to $a_{p}^{k-1}=m \cap a_{p}^{k}$. A similar contradiction arises if $x \nsupseteq r$; and since $a_{p}^{k-1} \nsupseteq r$, we must have $a_{p}^{k}>m \cap a_{p}^{k}$. Therefore either

$$
a_{p}^{k}>m \cap a_{p}^{k} \text { or } a_{q}^{n-k}>m \cap a_{q}^{n-k}
$$




$$
a_{p}^{k}>m \cap a_{p}^{k}>a_{p}^{k-1} \text { but } a_{q}^{n-k}>y>a_{q}^{n-k-1}=m \cap a_{q}^{n-k}
$$

As before, $v$ exists with $u>v, v \cap a_{p}^{k}=m \cap a_{p}^{k}$, and there is a chain from $m \cap a_{p}^{k}$ to $v$ of length at least $2 n-2 k$. There is a point $s \in C_{p}^{k}$ such that $s \nsubseteq m n a_{p}^{k}$, and hence $s \nsubseteq v$. Therefore $v \supseteq a_{q}^{n-k-1}$, so $v=m$. But $m / z$, by Theorem 1.1 , is equal to $m \cap a_{p}^{k} / z \vee a_{q}^{n-k-1 / z}$ in contradiction with the length of the chain from $m \cap a_{p}^{k}$ to $v=m$. Iíence $a_{q}^{n-k}>m \cap a_{q}^{n-k}$; and whenever $u>m \nsupseteq r$, we have

$$
a_{p}^{k}>m \cap a_{p}^{k}, a_{p}^{n-k}>m \cap a_{q}^{n-k}
$$

The converse of this theorem is not true; however we do have the following result:

THEOREM 3.2. If $a_{p}^{k}>x \supseteq r$, then $u>x \cup a_{q}^{n-k}$, while if $a_{q}^{n-k}>y \supseteq r$, then $u>a_{p}^{k} \cup y$. If $a_{p}^{k}>x \nsupseteq r$ and $a_{q}^{n-k}>y \nsupseteq r$, then $u>x \cup y$ if and only if for any points $t \subseteq x, s \subseteq y$, we have $t \cup s \nsupseteq r$.

Proof. Let $a_{p}^{k}>x \supseteq r$, and let $x^{\prime}$ be a relative complement of $a_{p}^{k}$ in $u / x$ such that $u>x^{\prime}$. Then by Theorem 3.1 we have $x^{\prime} \supseteq a_{q}^{n-k}$ and $x^{\prime}=x \cup a_{q}^{n-k}$. A similar argument shows that if $a_{q}^{n-k}>y \supseteq r$, then $u>a_{p}^{k} \cup y$.

Suppose

$$
a_{p}^{k}>x \nsupseteq r, a_{q}^{n-k} \supseteq y \nsupseteq r
$$

If

$$
x \supseteq t>z \text { and } y \supseteq s>z
$$

such that $s$ u $2 \supseteq r$, then

$$
x \cup y \supseteq r \text { and } x \cup y=(x \cup r) \cup(y \cup r)=u \text {. }
$$

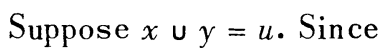

$$
x \nmid r, y \nmid r
$$

it follows that

$$
x \supseteq a_{p}^{k-1}, y \supseteq a_{q}^{n-k-1} .
$$

If $x=a_{p}^{k-1}$ or $y=a_{q}^{n-k-1}$, Lemma 2.2 tells us that $r \in C_{p}^{k}$ or $r \in C_{q}^{n-k}$. Hence

$$
x>a_{p}^{k-1} \text { and } y>a_{q}^{n-k-1} .
$$

So points $s$ and $t$ exist such that $x=t \cup a_{p}^{k-1}$ and $y=s \cup a_{q}^{n-k-1}$. Therefore 


$$
a_{p}^{k-1} \cup t \cup s \cup a_{q}^{n-k-1} \geq r
$$

and applying I emma 2.2 twice we get $t \cup s \supseteq r$. All that is required to finish the proof of the theorem is to show that if $u>m \supseteq x \cup y$, then $m=x \cup y$. Suppose $m \supseteq r$; then

$$
m \supseteq(x \cup r) \cup(y \cup r)=u
$$

So $m \nsupseteq r$. Hence, by Theorem 3.1, $m=x_{1} \cup y_{1}$, where

$$
a_{p}^{k}>x_{1} \ddagger r \text { and } a_{q}^{n-k}>y_{1} \ddagger r
$$

But this implies $x=x_{1}, y=y_{1}$, and $m=x \cup y$.

4. Maximal elements when $a_{p}^{k} \cap a_{q}^{n-k}=z$. Here as before we assume that $1 \leq k \leq n-1$.

THEOREM 4.1. If $u>m$ then $m$ is one of the following three types:

(1) $m \supseteq a_{p}^{k}, a_{q}^{n-k}>a_{q}^{n-k} \cap m \ddagger a_{q}^{n-k-1}$, or dually;

(2) $m \supseteq a_{p}^{k}, a_{q}^{n-k} \cap m=a_{q}^{n-k-1}$, or dually;

(3) $a_{p}^{k}>m \cap a_{p}^{k} \supseteq a_{p}^{k-1}$, and $a_{q}^{n-k}>m \cap a_{q}^{n-k} \supseteq a_{q}^{n-k-1}$.

Proof. Suppose

$$
u>m \supseteq a_{p}^{k}, m \neq a_{q}^{n-k-1} \text {, but } a_{q}^{n-k}>x \supset a_{q}^{n-k} \cap m .
$$

Then not all elements of $a_{q}^{n-k} / z$ covering $m \cap a_{q}^{n-k}$ will contain $a_{q}^{n-k-1}$. On the other hand,

$$
m=\left(m \cap a_{q}^{n-k}\right) \cup a_{p}^{k}
$$

so for any point

$$
s \subseteq a_{q}^{n-k}, s \nsubseteq m \cap a_{q}^{n-k}
$$

we have

$$
s \cup\left(m \cap a_{q}^{n-k}\right) \cup a_{p}^{k} \supseteq a_{q}^{n-k-1}
$$

Then by Lemma 2.2 we must have

$$
s \cup\left(m \cap a_{q}^{n-k}\right) \supseteq a_{q}^{n-k-1},
$$

contrary to the above assertion. 'Therefore if 


$$
u>m \supseteq a_{p}^{k} \text { and } m \nsupseteq a_{q}^{n-k-1}
$$

then $a_{q}^{n-k}>m \cap a_{q}^{n-k}$

Now suppose $u>m \supseteq a_{p}^{k}$ and $m \supseteq a_{q}^{n-k-1}$. If $m / z$ is simple, we contradict our maximal projectivity assumption; but arguing as before on the direct split of $m / z$, we see that

$$
m / z=a_{p}^{k} / z \vee a_{q}^{n-k-1} / z
$$

and hence $m \cap a_{q}^{n-k}=a_{q}^{n-k-1}$.

Finally suppose $u>m$, but $m \nsupseteq a_{p}^{k}, m \nsupseteq a_{q}^{n-k}$. Then $m \supseteq a_{p}^{k-1}$ and $m \supseteq a_{q}^{n-k-1}$. Assume $m \cap a_{p}^{k}=a_{p}^{k-1}$, and let $a_{p}^{k}>x>a_{p}^{k-1}$, by Lemma 2.3. Let $v$ be a relative complement of $a_{p}^{k}$ in $u / x$ such that $u>v$. Since $v \nsupseteq a_{p}^{k}$, we have $v \supseteq a_{q}^{n-k-1}$. Now $v \neq m$, so $a_{q}^{n-k}>m \cap a_{q}^{n-k}$. Then $m^{\prime}$ exists such that $u>m^{\prime}, m^{\prime} \neq a_{q}^{n-k}$, and there is a chain from $m \cap a_{q}^{n-k}$ to $m^{\prime}$ of length at least $2 k$. Since $m^{\prime} \notin a_{q}^{n-k}$, it follows that $m^{\prime} 2 a_{p}^{k-1}$, and hence $m^{\prime}=m$. But $m / z$ is not simple; $a_{p}^{k-1^{+}}$and $a_{q}^{n-k_{i}} n m$ are in different components. This is contrary to the length of the above chain, since $\rho\left(a_{p}^{k-1}\right)=2 k-1$. Hence we must have $a_{p}^{k}>m \cap a_{p}^{k}$, and dually $a_{q}^{n-k}>m \cap a_{q}^{n-k}$.

Examples show that it is impossible from the structures of $a_{p}^{k} / z$ and $a_{q}^{n-k} / z$ to tell whether $u>a_{p}^{k} \cup a_{q}^{n-k-1}$ or $u=a_{p}^{k} \cup a_{q}^{n-k-1}$, and dually. However, for the other maximal elements we have:

THEOREM 4.2. If

$$
a_{q}^{n-k}>y \neq a_{q}^{n-k-1},
$$

then $u>y \cup a_{p}^{k}$, and dually. If

$$
a_{p}^{k}>x \supseteq a_{p}^{k-1} \text { and } a_{q}^{n-k}>y \supseteq a_{q}^{n-k-1}
$$

then $u>x \cup y$ if and only if for every pair of points $s \subseteq x, t \subseteq y$ the lattice $s \cup t / z$ is a Boolean algebra.

Proof. Suppose

$$
a_{q}^{n-k}>y \supseteq a_{q}^{n-k-1} \text { and } u=a_{p}^{k} \cup y
$$

Then there is a point $t \subseteq a_{q}^{n-k-1}$ such that $t \nsubseteq y, t \nsubseteq a_{p}^{k}$, but $t \subseteq a_{p}^{k} \cup y$; and using I emma 2.2 we obtain a contradiction of our maximal projectivity hypothesis. On the other hand, if $u>m \supseteq a_{p}^{k} \cup y$, then by Theorem 4.1 we get $m=a_{p}^{k} \cup y$.

Let $a_{p}^{k}>x \supseteq a_{p}^{k-1}$ and $a_{q}^{n-k}>y \supseteq a_{q}^{n-k-1}$. By Theorem 4.1, either $u=x \cup y$ 
or $u>x \cup y$. If $u=x \cup y$, there are points $s \subseteq x, t \subseteq y$ such that

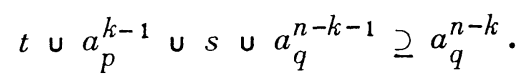

Then by Lemma 2.2, we have

$$
t \cup s \cup a_{q}^{n-k-1} \supseteq a_{q}^{n-k}
$$

thus $t \cup a_{q}^{n-k} / z$ is not a direct union, so there is another point $r \subseteq a_{p}^{k}$ such that $t \cup s \cup a_{q}^{n-k-1} \supseteq r$, and hence $t \cup s \supseteq r$. But this tells us that $t \cup s / z$ is not a Boolean algebra.

If $u>x \cup y$, we must have $x \cup y / z=x / z \vee y / z$, and the condition is satisfied.

Here again, then, save for the one exception, the structure of $L$ is determined by the structure of sublattices and the relations between points.

\section{References}

1. R. P. Dilworth, The structure of relatively complemented lattices, Ann. of Math. (2) 5 (1950), 348-359.

2. R. P. Dilworth and Morgan Ward, Note on paper by C. E. Rickart, Bull. Amer. Math. Soc. 55 (1949), 1141.

3. J. E. McLaughlin, Projectivities in relatively complemented lattices, Duke Math. J. 18 (1951), 73-84.

The University of Michigan 


\section{PACIFIC JOURNAL OF MATHEMATICS}

\section{EDITORS}

\author{
R. M. Foeinson \\ University of California \\ Berkeley 4, California \\ E. HEWITt \\ University of Washington \\ Seattle 5, Washington
}

R. P. DILWOR TH

California Institute of Technology

Pasadena 4, California

E. F. BECKENBACH

University of California

Los Angeles 24, California

\section{ASSOCIATE EDITORS}

$\begin{array}{llll}\text { H. BUSEMANN } & \text { P. R. HALMOS } & \text { BøRGE JESSEN } & \text { J. J. STOKER } \\ \text { HERBERT FFDERER } & \text { HEINZ HOPF } & \text { PAUL LÉVY } & \text { E. G. STR AUS } \\ \text { MARSHALL IIALI } & \text { R. D. JAMES } & \text { GEORGE PÓLYA } & \text { KỎSAKU YOSIDA }\end{array}$

\section{SPONSORS}

UNIVERSITY OF BRITISH COLUMBIA CAIIFORNIA INSTITUTE OF TECHNOLOGY UNIVERSITY OF CALIFORNIA, BERKELEY UNIVERSITY OF CAILIFORNIA, DAVIS UNIVERSITY OF CALIFORNIA, LOS ANGELES UNIVERSITY OF CALIFORNIA, SANTA BARBARA UNIVERSITY OF NEVADA OREGON STATE COLLEGE UNIVERSITY OF OREGON
UNIVERSITY OF SOUTHERN CALIFORNIA STANFORD RESEARCH INSTITUTE STANFORD UNIVERSITY WASHINGTON STATE COLLEGE UNIVERSITY OF WASHINGTON

AMERICAN MATHEMATICAL SOCIETY NATIONAL BUREAU OF STANDARDS, INSTITUTE FOR NUMERICAL ANALYSIS

$$
\begin{gathered}
\text { Vari-Type Composition by } \\
\text { Elaine Barth } \\
\text { Delores Wierman } \\
\text { With the cooperation of } \\
\text { E. F. Beckenbach } \\
\text { E. G. Straus }
\end{gathered}
$$

Printed in the United States of America by Edwards Brothers, Inc., Ann Arbor, Michigan 


\section{Pacific Journal of Mathematics}

\section{Vol. 3, No. 1 \\ March, 1953}

Herbert Busemann, Volume in terms of concurrent cross-sections ......... 1

L. Carlitz, Some special equations in a finite field ................. 13

Homer V. Craig and Billie Braden Townsend, On certain metric

extensors ....................................... 25

Philip J. Davis and Henry Pollak, Linear functionals and analytic

continuation problems ............................. 47

Jacob C. E. Dekker, The constructivity of maximal dual ideals in certain

Boolean algebras ................................. 73

Harley M. Flanders, The norm function of an algebraic field extension .... 103

Marshall Hall, Subgroups of free products . . . . . . . . . . . . . . . . . 115

Israel (Yitzchak) Nathan Herstein, Finite multiplicative subgroups in

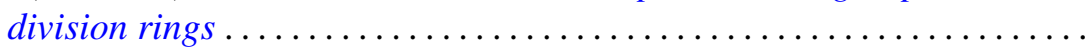

Joseph Lawson Hodges, Jr. and Murray Rosenblatt, Recurrence-time moments in random walks ............................ 127

Alfred Horn, The normal completion of a subset of a complete lattice and lattices of continuous functions ........................ 137

Fulton Koehler, Estimates for the errors in the Rayleigh-Ritz method...... 153

M. H. Martin, The Monge-Ampère partial differential equation

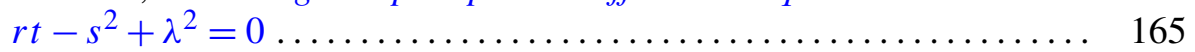

John E. Maxfield, Normal k-tuples ......................... 189

Jack E. McLaughlin, Structured theorems for relatively complemented lattices............................................ 197

William H. Mills, A system of quadratic Diophantine equations ......... 209

T. S. Motzkin, Ernst Gabor Straus and F. A. Valentine, The number of farthest points ................................. 221

G. Power, Forces on the boundary of a dielectric ............... 233

Ralph Gordon Selfridge, Approximations with least maximum error....... 247 